\title{
Copepod crustaceans parasitizing marine fish of the Kerala coast, India
}

\author{
Веслоногие ракообразные, паразитирующие на рыбе \\ прибрежкья штата Керала, Индия
}

\author{
R.M.V. Nikhila1, O.K. Drisya ${ }^{2}$, K. Rijin², T.M.V. Mumthaz ${ }^{2}$, A.K. Helna', \\ Viatcheslav N. Ivanenko ${ }^{3}$, Sudha Kappalli, ${ }^{1,2,}$

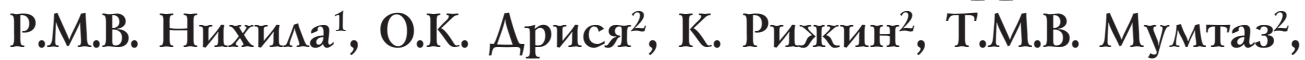 \\ А.К. Хелна, В.Н. Иваненко ${ }^{3}$, Судха Каппамли ${ }^{1,2, *}$
}

\footnotetext{
${ }^{1}$ Department of Animal Science, School of Biological Sciences, Central University of Kerala, Kasaragod, 671316, Kerala, India.

${ }^{2}$ Post Graduate Department of Zoology and Research Centre, Sree Narayana College, Kannur, 670007, Kerala, India.

${ }^{3}$ Department of Invertebrate Zoology, Faculty of Biology, Lomonosov Moscow State University, Moscow 119992, Russia.

${ }^{3}$ Кафедра зоологии беспозвоночных, Биологический факультет, Московский государственный университет им. М.В. Ломоносова, Москва 119992, Россия

*Corresponding author: sudhakappalli@cukerala.ac.in
}

KEY WORDS: parasitic copepod, Siphonostomatoida, Cyclopoida, fish, Kerala, India.

КЛЮЧЕВЫЕ СЛОВА: паразитические веслоногие ракообразные, сифоностоматоиды, циклопоиды, рыбы, Керала, Индия.

ABSTRACT: Copepods parasitizing marine fish were collected along the Cochin and Malabar coasts (Kerala, India) of the Indian Ocean from July 2013 through February 2019. One hundred species of copepods from 31 genera in eight families, were found living on 85 species fish represented 65 genera in 34 families. Copepods of the order Siphonostomatoida were the dominant parasites; they belonged to five families (Caligidae, Lernaeopodidae, Lernanthropidae, Pennellidae, and Pseudocycnidae), of which Caligidae and Lernanthropidae were the most diverse, comprising 29 species each (in eight and five genera, respectively). The genera Caligus and Lernanthropus, with 20 and 21 species, respectively, are the most diverse genera of siphonostomatoid copepods. All 26 species in nine genera of the poecilostome cyclopoids belong to the families Bomolochidae, Chondracanthidae and Ergasilidae. The great diversity of parasitic copepods living on fish suggests a need for a more thorough integrative study of the diversity, host specificity and ecology of these copepods, not only from fish along the Kerala coasts, but also along the other coasts of India.

How to cite this article: Nikhila R.M.V., Drisya O.K., Rijin K., Mumthaz T.M.V., Helna A.K., Ivanenko V.N., Kappalli S.2019. Copepod crustaceans parasitizing marine fish of the Kerala coast, India // Arthropoda Selecta. Vol.28. No.4. P.529-544. doi: 10.15298/ arthsel. 28.4.05

РЕЗЮМЕ: Копеподы, паразитирующие на морской рыбе, собирали в Индийском океане вдоль побережья Малабар и Кочин (штат Керала, Индия) с июля 2013 г. по февраль 2019 г. Выявлено сто видов копепод, относящихся к 31 роду восьми семейств, обитающих на 85 видах рыб, относящихся к 65 родами 34 семейств. Копеподы отряда Siphonostomatoida - преобладающие паразиты, относящиеся к пяти семействам: Caligidae, Lernaeopodidae, Lernanthropidae, Pennellidae и Pseudocycnidae. Семейства Caligidae и Lernanthropidae - наиболее разнообразные семейства, каждое из которых включает 29 видов и относящихся к восьми и пяти родам, соответственно. Роды Caligus и Lernanthropus, насчитывающие 20 и 21 видов - самые разнообразные рода сифоностоматоидных копепод. Все 26 видов девяти родов поецилостомовых циклопоид относятся к семействам Bomolochidae, Chondracanthidae и Ergasilidae. Большое разнообразие копепод, паразитирующих на рыбах, указывает на необходимость проведение тщательного комплексного исследования разнообразия, специфичности к хозяевам и экологии копепод не только вдоль побережья Кералы, но и у берегов других районов Индии.

\section{Introduction}

Marine fish host a great diversity of parasitic invertebrates, among which copepods are significant [Tripathi, 1960, 1962a,b; Silas, Ummerkutty, 1967; Kabata, 1985; Pillai, 1985]. However, a comprehensive account of this crustacean group along the coasts of India is still in its infancy despite the important initial work on parasitic copepods carried out before the mid-1980s. The first records of copepods parasitizing fish in Indi- 


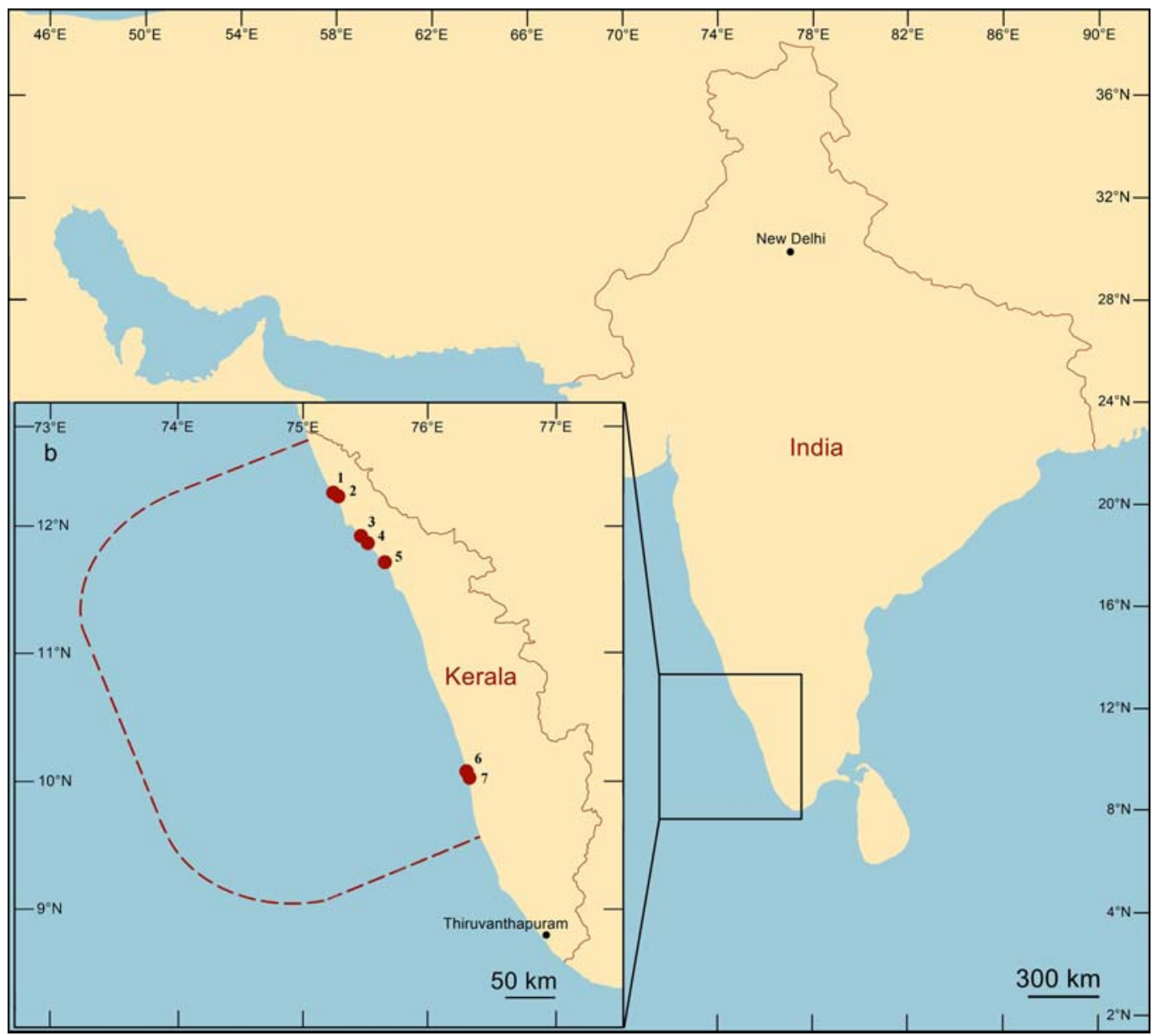

Fig. 1. Map of the fishing area and fisherman landing centres (1-7) at the Kerala coast of India; 1 - Thaikkadappuram, 2 Madakkara, 3 - Azhikkal, 4 - Ayikkara, 5 - Chombala, 6 - Kalamukku, 7 - Thoppumpadi.

Рис. 1. Карта района рыбного промысла в прибрежных водах и центры приема улова (1-7) в штате Керала, Индия; 1 Тайккадаппурам, 2 - Мадаккара, 3 - Ажиккал, 4 - Айиккара, 5 - Чомбала, 6 - Каламукку, 7 - Топпумпади.

an waters were contributed by Bassett-Smith [1898a,b; 1899], who described several copepods from the Arabian Sea. Gnanamuthu [1947a,b,c; 1948a, b; 1949; 1950a,b,c; 1951, 1953] and Rangnekar [1956, 1957, $1959,1960,1961]$ investigated the diversity of parasitic copepods in the Bay of Bengal and the Arabian Sea, respectively. Additional information on copepods infesting the fish of the Indian coast was published by Rao [1950], Kurian [1955, 1961], Tripathi [1962], Sebastain [1964, 1966], Sebastian \& George [1964], Bennet [1964, 1966, 1967], and Hameed \& Pillai [1973], and a review of the parasitic copepods living on fishes of India was published by Kirtisinghe [1964]. Later, Hameed [1976] described new species of copepods of the genera Lepeophtheirus and Lamproglena that were found on fish along the southern coast of Kerala. Pillai recorded and described 59 new species of copepods in the southern part of Kerala in a series of publications from 1961 through 1985 (see Pillai, 1985). More recent studies have reported copepods living on marine fish of the south eastern part of the Indian coast [Vinoth et al., 2010]. A description of ergasilid copepods on the fish of the estuarian and fresh waters of Kerala was presented by Ho et al. [1992] and Santhosh [2000].

An analysis of the literature shows that most records of the parasitic copepods of India focus on the most southern part of the country, with little attention paid to the middle and northern part of the Kerala coast. Studies of the diversity of parasitic copepods living on the fish of the Cochin and Malabar coasts of Kerala is limited to a few reports [Aneesh et al., 2013, 2014, 2018; Helna et al., 2013, 2016, 2018; Rijin et al., 2018]. The present paper represents the first review of the diversity of parasitic copepods living on the marine fish of the Cochin and Malabar coasts of Kerala, where we have collected since July 2013.

\section{Material and methods}

The fish were obtained from fishing boats at the landing centres of the Malabar (Ayikkara, Azhikkal, Chombala, Madakkara, and Thaikadappuram) and Cochin coasts (Kalamukku and Thoppumpadi) of Kerala from July 2013 through February 2019 (Fig. 1). 
The fishing range covered by the boats is marked in Figure 1. The fish collected at Ayyikkara, Thaikdappuram and Madakkara were caught approximately 25$30 \mathrm{~km}$ offshore in night fishing using gillnets. The fish obtained from other centres (Azhikkal, Chombala, Kalamukku, and Thoppumpadi) were caught approximately $250 \mathrm{~km}$ offshore using gill nets, trawl nets and trawl lines.

After sampling, the bodies of the fish (the surface, lateral line region, base of the pectoral fin, branchial cavity and gill filaments, inner wall of the operculum, etc.) were thoroughly examined for the presence of parasitic copepods using a hand lens. The infected fish were then brought to the laboratory, where the copepods were removed from the fish under a dissection microscope (Leica Stereo zoom S6-D, Lieca Stereo zoom LAS X) and preserved in $70 \%$ ethanol. The identification of the copepods was conducted with the identification keys [Yamaguti, 1963; Kabata, 1985; Pillai, 1985]. Original descriptions of the species were consulted whenever possible. The updated taxonomy of the copepods and their hosts was obtained from the WoRMS database [WoRMS, 2019], and the taxonomical identification of the fish hosts was made according to the FishBase [Froese, Pauly, 2019]. The collected copepods were stored at the Crustacean Research Laboratory of the Central University of Kerala, India.

\section{Results}

One hundred copepod species were found on 85 species of fish belonging to 65 genera and 34 families distributed along the Cochin and Malabar coasts of Kerala, India (Table 1, Fig. 2). Seventy-four species belong to the order Siphonostomatoida in the following families (with the number of species and genera): Caligidae (29 species in eight genera), Lernanthropidae (29 species in five genera), Lernaeopodidae (12 species in five genera), Pseudocycnidae (two species in two genera) and Pennellidae (two species in two genera). Among the genera, Caligus (Caligidae) and

Table 1. Marine fish-hosts of parasitic copepods from the Kerala coast of India. Таблица 1. Морские рыбы - хозяева паразитических копепод из прибрежных вод штата Керала, Индия.

\begin{tabular}{|c|c|c|}
\hline $\begin{array}{l}\text { Fish-host family } \\
\text { name }\end{array}$ & Fish-host species name & Parasitic copepod species name \\
\hline Acanthuroidae & Acanthurus mata (Cuvier, 1829) & Nothobomolochus trichiuri \\
\hline \multirow{3}{*}{ Ariidae } & \multirow{2}{*}{ Arius acutirostris Day, 1877} & Caligus arii \\
\hline & & Hermilius pyriventris \\
\hline & Netuma thalassina (Rüppell, 1837) & H. pyriventris \\
\hline \multirow{12}{*}{ Belonidae } & Ablennes hians (Valenciennes, 1846) & Lernanthropus belones \\
\hline & Platybelone argalus (Lesueur, 1821) & Nothobomolochus gibber \\
\hline & $\begin{array}{c}\text { Strongylura incisa (Valenciennes, } \\
1846)\end{array}$ & N. gibber \\
\hline & \multirow{5}{*}{ Strongylura leiura (Bleeker, 1850) } & Bomolochus bellones \\
\hline & & Caligodes laciniatus \\
\hline & & Lernanthropus belones \\
\hline & & L. tylosuri \\
\hline & & Nothobomolochus gibber \\
\hline & \multirow{4}{*}{ S. strongylura (van Hasselt, 1823) } & Bomolochus bellones \\
\hline & & Dermoergasilus coleus \\
\hline & & Lernanthropus tylosuri \\
\hline & & Nothobomolochus gibber \\
\hline \multirow{12}{*}{ Carangidae } & \multirow{4}{*}{ Alepes djedaba (Forsskål, 1775) } & Bomolochus decapteri \\
\hline & & Caligus constrictus \\
\hline & & Lernanthropus corniger \\
\hline & & L. giganteus \\
\hline & A. kleinii (Bloch, 1793) & Caligus bonito \\
\hline & $\begin{array}{c}\text { Carangoides coeruleopinnatus } \\
\text { (Rüppell, 1830) }\end{array}$ & Lernanthropus koenigii \\
\hline & Caranx ignobilis (Forsskål, 1775) & Caligus rotundigenitalis \\
\hline & \multirow{2}{*}{ C. sexfasciatus Quoy et Gaimard, 1825} & Bomolochus multiceros \\
\hline & & Lernanthropus indicus \\
\hline & \multirow{3}{*}{ Decapterus russelli (Rüppell, 1830) } & Bomolochus selaroides \\
\hline & & Caligus kanagurta \\
\hline & & Lernanthropinus decapteri \\
\hline
\end{tabular}


Table 1 (continued). Таблица 1 (продолжение).

\begin{tabular}{|c|c|c|}
\hline $\begin{array}{l}\text { Fish-host family } \\
\text { name }\end{array}$ & Fish-host species name & Parasitic copepod species name \\
\hline \multirow{8}{*}{ Carangidae } & \multirow{2}{*}{ Megalaspis cordyla (Linnaeus, 1758) } & Caligus cordyla \\
\hline & & Lernanthropus corniger \\
\hline & \multirow{4}{*}{ Parastromateus niger (Bloch, 1795) } & Bomolochus megaceros \\
\hline & & Lernaeenicus stromatei \\
\hline & & Synestius caliginus \\
\hline & & Thysanote appendiculata \\
\hline & Scomberoides tol (Cuvier, 1832) & Bomolochus decapteri \\
\hline & $\begin{array}{c}\text { Selar crumenophthalmus (Bloch, } \\
1793 \text { ) }\end{array}$ & B. selaroides \\
\hline Cichlidae & Etroplus suratensis (Bloch, 1790) & Caligus rotundigenitalis \\
\hline \multirow{9}{*}{ Clupeidae } & \multirow{3}{*}{$\begin{array}{c}\text { Anodontostoma chacunda (Hamilton, } \\
\text { 1822) }\end{array}$} & Naobranchia cygniformis \\
\hline & & Peniculus fistula fistula \\
\hline & & Pseudorbitacolax varunae \\
\hline & $\begin{array}{c}\text { Escualosa thoracata (Valenciennes, } \\
1847 \text { ) }\end{array}$ & Ergasilus parvitergum \\
\hline & \multirow{4}{*}{$\begin{array}{c}\text { Sardinella fimbriata (Valenciennes, } \\
1847)\end{array}$} & Clavellisa dussumieriae \\
\hline & & C. ilishae \\
\hline & & Mitrapus oblongus \\
\hline & & Pumiliopes squamosus \\
\hline & S. longiceps Valenciennes, 1847 & Clavellisa ilishae \\
\hline Coryphaenidae & Coryphaena hippurus Linnaeus, 1758 & Euryphorus nordmannii \\
\hline Dussumieriidae & $\begin{array}{c}\text { Dussumieria acuta Valenciennes, } \\
1847 \\
\end{array}$ & Lernanthropus dussumieria \\
\hline \multirow{10}{*}{ Engraulidae } & Coilia dussumieri Valenciennes, 1848 & Clavellisa phasa \\
\hline & $\begin{array}{c}\text { Stolephorus baganensis Hardenberg, } \\
1933\end{array}$ & Mitrapus engraulis \\
\hline & S. tri (Bleeker, 1852) & M. engraulis \\
\hline & \multirow{4}{*}{ Thryssa malabarica (Bloch, 1795) } & Bomolochus multiceros \\
\hline & & Clavellisa obcordatus \\
\hline & & Lernanthropinus decapteri \\
\hline & & Nothobomolochus multispinosus \\
\hline & \multirow{2}{*}{ T. mystax (Bloch et Schneider, 1801) } & Clavellisa obcordatus \\
\hline & & Nothobomolochus multispinosus \\
\hline & T. setirostris (Broussonet, 1782) & Clavellisa obcordatus \\
\hline \multirow{2}{*}{ Gerreidae } & Gerres erythrourus (Bloch, 1791) & Lernanthropus triangularis \\
\hline & G. filamentosus Cuvier, 1829 & Nothobomolochus gerresi \\
\hline \multirow{6}{*}{ Hemiramphidae } & Hemiramphus far (Forsskål, 1775) & Bomolochus cuneatus \\
\hline & \multirow{4}{*}{ H. lutkei Valenciennes, 1847} & B. bellones \\
\hline & & B. cuneatus \\
\hline & & Lernanthropus tylosuri \\
\hline & & Nothobomolochus denticulatus \\
\hline & $\begin{array}{c}\text { Rhynchorhamphus malabaricus } \\
\text { Collette, } 1976\end{array}$ & N. cypseluri \\
\hline Labridae & $\begin{array}{c}\text { Parajulis poecilepterus (Temminck et } \\
\text { Schlegel,1845) }\end{array}$ & Caligus triangularis \\
\hline \multirow{4}{*}{ Leiognathidae } & Equulites elongatus (Günther, 1874) & Lernanthropus leiognathi \\
\hline & Eubleekeria splendens (Cuvier, 1829) & L. leiognathi \\
\hline & \multirow{2}{*}{$\begin{array}{l}\text { Leiognathus brevirostris } \\
\text { (Valenciennes, 1835) }\end{array}$} & L. leiognathi \\
\hline & & Nothobomolochus quadriceros \\
\hline
\end{tabular}


Table 1 (continued). Таблица 1 (продолжение).

\begin{tabular}{|c|c|c|}
\hline $\begin{array}{l}\text { Fish-host family } \\
\text { name }\end{array}$ & Fish-host species name & Parasitic copepod species name \\
\hline \multirow{7}{*}{ Leiognathidae } & Leiognathus daura (Cuvier, 1829) & Lernanthropus leiognathi \\
\hline & L. equulus (Forsskål, 1775) & L. leiognathi \\
\hline & L. fasciatus (Lacepède, 1803) & L. leiognathi \\
\hline & $\begin{array}{c}\text { Nuchequula blochii (Valenciennes, } \\
1835)\end{array}$ & L. leiognathi \\
\hline & $\begin{array}{c}\text { Photopectoralis bindus (Valenciennes, } \\
1835)\end{array}$ & L. leiognathi \\
\hline & Secutor insidiator $($ Bloch, 1787$)$ & L. secutoris \\
\hline & S. ruconius (Hamilton, 1822) & L. secutoris \\
\hline Lutjanidae & Lutjanus sanguineus (Cuvier, 1828) & L. brevicephalus \\
\hline Megalopidae & $\begin{array}{l}\text { Megalops cyprinoides (Broussonet, } \\
1782 \text { ) }\end{array}$ & L. cadenati \\
\hline Menidae & $\begin{array}{c}\text { Mene maculata (Bloch et Schneider, } \\
1801)\end{array}$ & Lernanthropinus sphyraenae \\
\hline \multirow{4}{*}{ Mugilidae } & $\begin{array}{c}\text { Gracilimugil argenteus (Quoy et } \\
\text { Gaimard, 1825) }\end{array}$ & Nothobomolochus digitatus \\
\hline & \multirow{3}{*}{ Mugil cephalus Linnaeus, 1758} & Bomolochus nitidus \\
\hline & & Caligus rotundigenitalis \\
\hline & & Ergasilus rostralis \\
\hline Nemipteridae & Scolopsis bimaculate Rüppell, 1828 & Mitrapus oblongus \\
\hline \multirow{5}{*}{ Polynemidae } & \multirow{3}{*}{$\begin{array}{l}\text { Eleutheronema tetradactylum (Shaw, } \\
1804)\end{array}$} & Lernanthropus polynemi \\
\hline & & Parapetalus hirsutus \\
\hline & & Thysanote eleutheronema \\
\hline & \multirow{2}{*}{$\begin{array}{c}\text { Leptomelanosoma indicum (Shaw, } \\
1804 \text { ) }\end{array}$} & Caligus longicaudatus \\
\hline & & Parapetalus hirsutus \\
\hline \multirow{2}{*}{ Priacanthidae } & \multirow{2}{*}{ Priacanthus hamrur (Forsskål, 1775) } & Caligus priacanthi \\
\hline & & Norion priacanthi \\
\hline \multirow{3}{*}{ Pristigasteridae } & \multirow{2}{*}{ Opisthopterus tardoore (Cuvier, 1829) } & Lernanthropus ilishae \\
\hline & & L. opisthopteri \\
\hline & Pellona ditchela Valenciennes, 1847 & Clavellisa obcordatus \\
\hline \multirow{2}{*}{ Psettodidae } & \multirow{2}{*}{$\begin{array}{l}\text { Psettodes erumei (Bloch et Schneider, } \\
1801)\end{array}$} & Ergasilus parvitergum \\
\hline & & Protochondracanthus alatus \\
\hline Scatophagidae & Scatophagus argus (Linnaeus, 1766) & Caligus rotundigenitalis \\
\hline \multirow{9}{*}{ Sciaenidae } & Johnius glaucus (Day, 1876) & Lernanthropus sciaeni \\
\hline & J. trachycephalus (Bleeker, 1851) & L. sciaeni \\
\hline & $\begin{array}{l}\text { Nibea maculata (Bloch et Schneider, } \\
1801)\end{array}$ & L. abitocephalus \\
\hline & \multirow{2}{*}{ Otolithes cuvieri Trewavas, 1974} & L. latis \\
\hline & & Parabrachiella otolithi \\
\hline & \multirow{4}{*}{$\begin{array}{l}\text { Otolithes ruber (Bloch et Schneider, } \\
1801)\end{array}$} & Bomolochus multiceros \\
\hline & & Brachiella thynni \\
\hline & & Lernanthropus otolithi \\
\hline & & Parabrachiella albida \\
\hline \multirow{9}{*}{ Scombridae } & $\begin{array}{c}\text { Auxis thazard thazard (Lacepède, } \\
1800)\end{array}$ & Caligus biseriodentatus \\
\hline & \multirow{8}{*}{ Euthynnus affinis (Cantor, 1849) } & C. amblygenitalis \\
\hline & & C. asymmetricus \\
\hline & & C. bonito \\
\hline & & C. coryphaenae \\
\hline & & C. cybii \\
\hline & & C. pelamydis \\
\hline & & C. regalis \\
\hline & & Pseudocycnus appendiculatus \\
\hline
\end{tabular}


Table 1 (continued). Таблица 1 (продолжение).

\begin{tabular}{|c|c|c|}
\hline $\begin{array}{l}\text { Fish-host family } \\
\text { name }\end{array}$ & Fish-host species name & Parasitic copepod species name \\
\hline \multirow{15}{*}{ Scombridae } & \multirow{6}{*}{ Rastrelliger kanagurta (Cuvier, 1816) } & Caligus kanagurta \\
\hline & & Ceratocolax euthynni \\
\hline & & Naobranchia vermiformis \\
\hline & & Nothobomolochus kanagurta \\
\hline & & Orbitacolax aculeatus \\
\hline & & Pumiliopes jonesi \\
\hline & $\begin{array}{c}\text { Sarda orientalis }(\text { Temminck et } \\
\text { Schlegel, 1844) }\end{array}$ & Caligus pelamydis \\
\hline & \multirow{6}{*}{$\begin{array}{c}\text { Scomberomorus commerson } \\
\text { (Lacepède, 1800) }\end{array}$} & C. bonito \\
\hline & & C. cybii \\
\hline & & C. infestans \\
\hline & & C. phipsoni \\
\hline & & Cybicola armatus \\
\hline & & Tuxophorus cybii \\
\hline & Thunnus albacores (Bonnaterre,1788) & Pseudocycnus appendiculatus \\
\hline & T. tonggol (Bleeker, 1851) & P. appendiculatus \\
\hline Serranidae & $\begin{array}{l}\text { Epinephelus diacanthus } \\
\text { (Valenciennes, 1828) }\end{array}$ & Sagum epinepheli \\
\hline Sillaginidae & Sillago sihama (Forsskål, 1775) & Lernanthropus sillaginis \\
\hline \multirow[t]{2}{*}{ Sparidae } & \multirow[t]{2}{*}{$\begin{array}{c}\text { Argyrops filamentosus (Valenciennes, } \\
1830 \text { ) }\end{array}$} & L. triangularis \\
\hline & & Nothobomolochus multispinosus \\
\hline \multirow{3}{*}{ Sphyraenidae } & Sphyraena barracuda (Edwards, 1771) & N. denticulatus \\
\hline & S. jello Cuvier, 1829 & N. denticulatus \\
\hline & S. obtusata Cuvier, 1829 & N. denticulatus \\
\hline \multirow{2}{*}{ Stromateidae } & \multirow{2}{*}{ Pampus argenteus (Euphrasen, 1788) } & Caligus stromatei \\
\hline & & Nothobomolochus triceros \\
\hline \multirow{3}{*}{ Synodontidae } & \multirow{2}{*}{ Saurida tumbil (Bloch, 1795) } & Abasia platyrostris \\
\hline & & Lernanthropinus temminckii \\
\hline & S. undosquamis (Richardson, 1848) & L.s temminckii \\
\hline Terapontidae & $\begin{array}{l}\text { Rhynchopelates oxyrhynchus } \\
\text { (Temminck et Schlegel, 1842) }\end{array}$ & L. villiersi \\
\hline \multirow{7}{*}{ Trichiuridae } & \multirow{3}{*}{ Lepturacanthussavala (Cuvier, 1829) } & Brachiella trichiuri \\
\hline & & Lernanthropinus forficatus \\
\hline & & Nothobomolochus trichiuri \\
\hline & \multirow{4}{*}{ Trichiurus lepturus Linnaeus, 1758} & Brachiella trichiuri \\
\hline & & Caligus savala \\
\hline & & Lernanthropinus forficatus \\
\hline & & Nothobomolochus trichiuri \\
\hline \multirow[b]{2}{*}{ Rachycentridae } & \multirow[b]{2}{*}{$\begin{array}{c}\text { Rachycentron canadum (Linnaeus, } \\
1766)\end{array}$} & Parapetalus occidentalis \\
\hline & & Tuxophorus caligodes \\
\hline
\end{tabular}

Lernanthropus (Lernanthropidae) were represented by 20 and 21 species, respectively (or 69 and $72 \%$ of the total discovered species of caligids and lernanthropids, respectively). Cyclopoid copepods (26 species) belonged to nine genera in the families Bomolochidae, Chondracanthidae and Ergasillidae, of which the most diverse genera, Bomolochus and Nothobomolocus, include seven and 10 species, respectively. The checklist includes the synonymised names of the collected copepods and their hosts along with the previous records from the Indian coast. The great diversity of parasitic copepods and the recording of 91 new records of hosts 


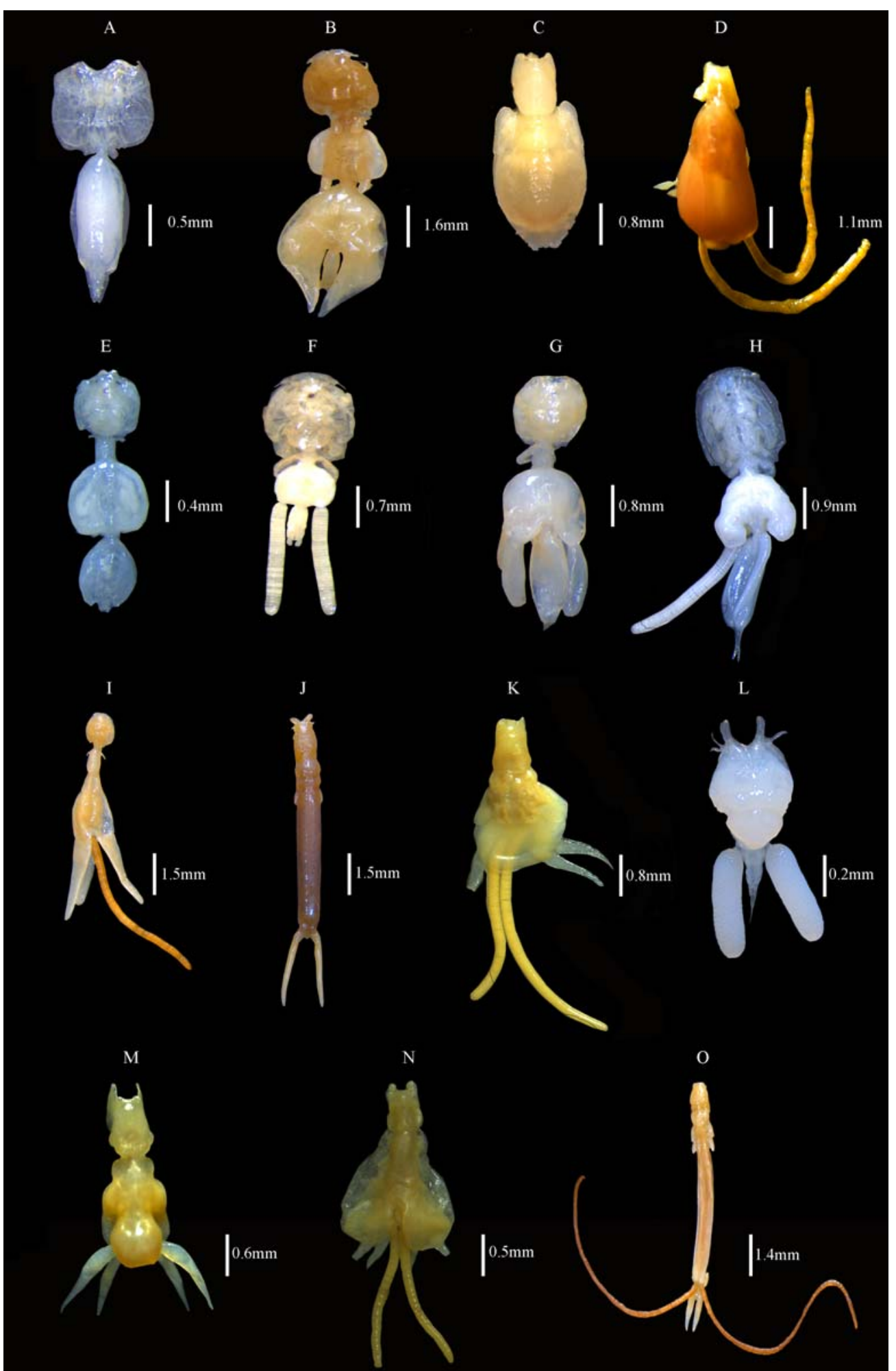

Fig. 2. Habitus of parasitic copepods infecting fish of the Kerala coast. A - Hermilius pyriventris from Netuma thalassina, B Euryphorus nordmannii from Coryphaena hippurus, C - Norion priacanthi from Priacanthus hamrur, D - Lernanthropus tylosuri from Strongylura leiura, E - Abasia platyrostris from Saurida tumbil, F - Caligus rotundigenitalis from Etroplus suratensis, G Synestius caliginus from Parastromateus niger, $\mathrm{H}$ - Parapetalus hirsutus from Eleutheronema tetradactylum, I — Caligodes laciniatus from Strongylura leiura, J - Pseudocycnus appendiculatus from Euthynnus affinis, K - Lernanthropus giganteus from Alepes djedaba, L - Nothobomolochus kanagurta from Rastrelliger kanagurta, M - Lernanthropus corniger from Megalaspis cordyla, $\mathrm{N}$ - Lernanthropinus sphyraenae from Mene maculata, O - Cybicola armatus from Scomberomorus commerson.

Рис. 2. Внешний вид паразитических копепод, заражающих рыбу в прибрежных водах штата Керала. 
for 56 species of copepods demonstrate the need for a thorough, integrative study of the host specificity, diversity and ecology of these copepods from Kerala and other parts of India.

\section{List of copepods parasitizing marine fish of the}

\section{Cochin and Malabar coasts}

(“*” marks host of copepod previously unknown in India; "f" marks fish marked in coral communities)

Subclass Copepoda Milne Edwards, 1830

Order Cyclopoida Burmeister, 1834

Family Bomolochidae Claus, 1875

Genus Bomolochus von Nordmann, 1832

Bomolochus bellones Burmeister, 1833

Material: 14 우, North Malabar (Ayikkara), 13.05.2016, on Strongylura leiura:; 13 + on S. strongylura; 2 우, North Malabar (Madakkara), 30.10.2018, on S. leiurat; 117 우, Cochin (Kalamukku), 6.05.2015, on Hemiramphus lutkei*

Records in India: South Kerala (Vizhinjam) [Pillai, Natarajan, 1977]; North Malabar (Ayikkara) [Aneesh, 2014]; on Ablennes hians, Belone belone, B. svetovidovi, Platybelone argalus, Strongylura anastomella, S. incisa, S. leiura, S. marina, S. strongylura, S. timucu, S. urvillii, Tylosurus acus, and T. crocodilus.

Bomolochus cuneatus Fraser, 1920

Material: 11 우, North Malabar (Azhikkal), 24.07.2013, on Hemiramphus lutkei (Valenciennes, 1847)*; 5 +9 , North Malabar (Azhikkal), 29.03.2014, on H. far (Forsskål, 1775)*ł.

Bomolochus decapteri Yamaguti, 1936

Material: 74 9ᄋ, North Malabar (Ayikkara), 8.04.2014, on Alepes djedaba*\$; 16 우, North Malabar (Ayikkara), 8.04.2014,on Scomberoides tol (Cuvier, 1832)*\$; 55 우, Cochin (Thoppumpadi) 14.12 .2017 , on $A$. djedaba*.

Records in India: South Kerala (Vizhinjam) [Pillai, 1967]; North Malabar (Ayikkara) [Aneesh, 2014]; on Carangoides malabaricus.

Bomolochus megaceros Heller, 1865

Material: 138 우, North Malabar (Ayikkara), 8.02.2014; 113 우, Cochin (Kalamukku), 15.12.2015, 32 우, Cochin (Thoppumpadi), 13.07.2015, all on Parastromateus nigert.

Records in India: Bombay [Basset-Smith, 1898a]; Chennai [Gnanamuthu, 1949]; South Kerala (Vizhinjam) [Pillai, 1967]; North Malabar (Ayikkara) [Aneesh, 2014]; on Alepes djedaba tromateus niger, and Terapon theraps.

Bomolochus multiceros Pillai et Natarajan, 1977 Material: 2 우, North Malabar (Ayikkara), 10.09.2016 on Caranx sexfasciatus $*$ t; 50 우, Cochin (Kalamukku), 26.03.2015, on Thryssa malabarica*; 108 우, Cochin (Kalamukku), 2.06.2015, on Otolithes ruber*.

Records in India: South Kerala (Vizhinjam) [Pillai, Natarajan, 1977], on Ariomma indicum.

Bomolochus nitidus Wilson C.B., 1911

Material: 6 우, North Malabar (Azhikkal), 2.08.2015; 10 오, North Malabar (Ayikkara), 16.10.2015, all on Mugil cephalus.

Records in India: North Malabar (Ayikkara) [Aneesh, 2014], on M. cephalus.
Bomolochus selaroides Pillai, 1965

Material: 3 우, North Malabar (Ayikkara), 17.08.2016, on Selar crumenophthalmus*; 32 +o, Cochin (Thoppumpadi); 4.02. 2016, on Decapterus russelli*.

Records in India: South Kerala (Vizhinjam) [Pillai, 1967], on Selaroides leptolepis.

Genus Ceratocolax Vervoort, 1965

Ceratocolax euthynni Vervoort, 1965

Material: $12 \mathrm{O}^{7} \mathrm{O}^{7}$, North Malabar (Ayikkara), 8.10.2014, on Rastrelliger kanagurta*.

Genus: Nothobomolochus Vervoort, 1962

Nothobomolochus cypseluri (Yamaguti, 1953)

Material: 3 우, North Malabar (Ayikkara), 12.12.2014, on Rhynchorhamphus malabaricus*.

Records in India: South Kerala (Vizhinjam) [Pillai, Natarajan, 1977], on Cheilopogon agoo. 1898)

Nothobomolochus denticulatus (Bassett-Smith,

Material: 35 우, North Malabar (Ayikkara), 7.10.2015, on Sphyraena barracuda*; 65 우, 7.10.2015, on S. obtusata*; 77 우, Cochin (Kalamukku), 17.12.2015, on S. jello; 35 우, Cochin (Kalamukku), 12.05.2015, on Hemiramphus lutkei*广.

Records in India: South Kerala (Trivandrum) [Gnanamuthu, 1947c; Pillai, 1967], on S. jello; North Malabar (Ayikkara) [Aneesh, 2014], on Hemiramphus far.

Nothobomolochus digitatus Cressey in Cressey et Collette, 1970

Material: 9 우, North Malabar (Ayikkara), 13.06.2013, on Gracilimugil argenteus*.

Nothobomolochus gerresi Pillai, 1973

Material: 44 우, North Malabar (Ayikkara), 16.03.2018; 39 우 (Kalamukku), 18.11.2015, all on Gerres filamentosus.

Records in India: South Kerala (Vizhinjam) [Pillai, 1973], on G. filamentosus.

Nothobomolochus gibber (Shiino, 1957)

Material: 1 , North Malabar (Azhikkal), 5.11.2014, on Platybelone argalus \$; 2 우, North Malabar (Azhikkal), 29.03.2014, on Strongylura incisa*; 1 , North Malabar (Azhikkal), 8.07.2013, on S. strongylura*; 1 q, North Malabar (Azhikkal), 8.04.2014, on $S$. leiura*†.

Records in India: South Kerala (Vizhinjam) [Pillai, Natarajan, 1977]; on Ablennes hians, Belone belone, B. svetovidovi, Platybelone argalus, Tylosurus acus, and T. crocodilus.

Nothobomolochus kanagurta (Pillai, 1965)

Material: 22 우, North Malabar (Ayikkara), 16.05.2016; 5 우, North Malabar (Thaikadappuram), 24.02.2018; 17 우, North Malabar (Madakkara), 18.04.2018; 24 오, Cochin (Kalamukku), 5.10.2016; 19 우, Cochin (Thoppumpadi), 22.10.2016, all on Rastrelliger kanagurta.

Records in India: South Kerala (Vizhinjam) [Pillai, 1967]; North Malabar (Ayikkara) [Aneesh, 2014], on R. kanagurta. 1949)

Nothobomolochus multispinosus (Gnanamuthu,

Material: 1 , , North Malabar (Ayikkara), 17.03.2015, on Thryssa malabarica*; 4 우, North Malabar (Azhikkal), 27.01.2017, on 
T. mystax*; 1 , North Malabar (Azhikkal), 27.01.2017, on Argyrops filamentosus $*$.

Records in India: South Kerala (Vizhinjam) [Gnanamuthu, 1947c, Chennai; Pillai, 1967d], on Dussumieria acuta and Dussumieria elopsoides.

Nothobomolochus quadriceros Pillai, 1973

Material: 22 우, North Malabar (Ayikkara), 20.06.2013, on Leiognathus brevirostris*.

Records in India: South Kerala (Vizhinjam) [Pillai, 1973], on Gazza minuta.

Nothobomolochus triceros (Bassett-Smith, 1898)

Material: 133 우, Cochin (Kalamukku), 18.03.2015; 38 우, Cochin (Thoppumpadi) 2.06.2015, all on Pampus argenteus.

Records in India: Bombay [Basset-Smith, 1899]; South Kerala, Trivandrum [Gnanamuthu, 1947c, Chennai; Pillai, 1967]; North Malabar (Ayikkara) [Aneesh, 2014], on $P$. argenteus.

Nothobomolochus trichiuri Pillai et Natarajan, 1977

Material: 11 우, North Malabar (Ayikkara), 4.04.2016, on Lepturacanthus savala; 18 오, North Malabar (Ayikkara), 4.04 2016, on Trichiurus lepturus; 2 우, North Malabar (Ayikkara), 17.11.2016, on Acanthurus mata*.

Records in India: South Kerala (Vizhinjam) [Pillai, Natarajan, 1977], on L. savala.

\section{Genus Orbitacolax Shen, 1957}

Orbitacolax aculeatus (Pillai, 1962)

Material: 30 우, North Malabar (Ayikkara), 25.04.2014, on Rastrelliger kanagurta.

Records in India: South Kerala (Vizhinjam) [Pillai, 1967], on R. kanagurta.

\section{Genus Pseudorbitacolax Pillai, 1971}

Pseudorbitacolax varunae (Bennet, 1968)

Material: $188 \bigcirc \bigcirc$, North Malabar (Ayikkara), 6.04.2015; 2 우, North Malabar (Thaikadappuram), 2.02.2018; 12 우, North Malabar (Madakkara), 4.09.2018; 147 우, Cochin (Kalamukku), 14.07.2015, all on Anodontostoma chacunda.

Records in India: South Kerala (Kochi) [Bennet, 1966; Pillai, 1971], on A. chacunda.

\section{Genus Pumiliopes Shen, 1957}

Pumiliopes jonesi (Bennet, 1968)

Material: 1002 우, North Malabar (Ayikkara), 2.06.2014, on Rastrelliger kanagurta.

Records in India: Malabar, Calicut [Bennet, 1967], on R. kanagurta.

Pumiliopes squamosus Cressey \& Boyle, 1973

Material: 12 우, North Malabar (Ayikkara), 14.05.2015 on Sardinella fimbriata.

Records in India: North Malabar (Ayikkara) [Aneesh, 2014], on S. fimbriata.

Family Chondracanthidae Milne Edwards, 1840 Genus Protochondracanthus Kirtisinghe, 1950

Protochondracanthus alatus (Heller, 1865)

Material: 4 우, North Malabar (Ayikkara), 14.07.2018, on Psettodes erumei.
Records in India: Bombay [Basset-Smith, 1898a]; South Kerala (Vizhinjam) [Pillai, 1967]; on P. erumei.

Genus Dermoergasilus Ho et Do, 1982

Dermoergasilus coleus (Cressey in Cressey et Collette, 1970)

Material: 3 우 North Malabar (Ayikkara), 8.07.2013, on Strongylura strongylura*.

Family Ergasilidae Burmeister, 1835

Genus Ergasilus Nordmann, 1832

Ergasilus parvitergum Ho, Jayarajan et Radhakrishnan, 1992

Material: 9 우, Cochin (Kalamukku), 7.08.2017, on Psettodes erumei*; 37 우, Cochin (Kalamukku), 9.10.2017, on Escualosa thoracata*.

Records in India: South Kerala [Ho et al., 1992], on Carangoides malabaricus and Etroplus suratensis.

Ergasilus rostralis Ho, Jayarajan et Radhakrishnan, 1992

Material: 140 우, Cochin (Kalamukku), 18.06.2015, on Mugil cephalus*.

Records in India: South Kerala [Ho et al., 1992], on Liza macrolepis, L. tade and Valamugil seheli.

Order Siphonostomatoida Thorell, 1859

Family Caligidae Burmeister, 1835

Genus Abasia Wilson C.B., 1908

Abasia platyrostris Pillai, 1963

Material: 21 우, North Malabar (Madakkara), 12.05.2018, on Saurida tumbil.

Records in India: South Kerala (Vizhinjam) [Pillai, 1967], on $S$. tumbil and Synodus indicus.

\section{Genus Caligodes Heller, 1865}

Caligodes laciniatus (Krøyer, 1863)

Material: 28 우, North Malabar (Ayikkara), 4.11.2017, on Strongylura leiura

Records in India: Indian coast [Rangnekar, 1959; Pillai, 1961], on Ablennes hians, Platybelone argalus argalus, Strongylura leiura, S. strongylura, Tylosurus acus, T. choram, and T. crocodilus; North Malabar (Ayikkara) [Aneesh, 2014], on S. strongylura.

\section{Genus Caligus Müller, 1785}

Caligus amblygenitalis Pillai, 1961

Material: 9 우, North Malabar (Ayikkara), 8.10.2014, on Euthynnus affinis.

Records in India: South Kerala (Vizhinjam) [Pillai, 1967]; Indian coast [Silas, Ummerkutty 1967], on E. affinis.

Caligus arii Bassett-Smith, 1898

Material: 12 우, North Malabar (Ayikkara), 11.10.2013 on Arius acutirostris.

Records in India: North Malabar (Ayikkara) [Aneesh, 2014], on A. acutirostris; South Kerala (Vizhinjam) [Pillai, 1970], on A. acutirostris and Plicofollis dussumieri. 
Caligus asymmetricus Kabata, 1965

Material: 14 우, North Malabr (Ayikkara), 14.11.2014, on Euthynnus affinis.

Records in India: South Kerala (Vizhinjam) [Pillai, 1967], on E. affinis, E. alletteratus, Grammatorcynus bilineatus, and Sarda orientalis.

\section{Caligus biseriodentatus Shen, 1957}

Material: 8 우, North Malabar (Ayikkara), 13.02.2015, on Auxis thazard.

Records India: South Kerala (Vizhinjam) [Pillai, 1967]; North Malabar (Ayikkara) [Silas, Ummerkutty, 1967; Aneesh, 2014], on A. thazard.

\section{Caligus bonito Wilson, 1905}

Material: 4 우, North Malabar (Ayikkara), 17.08.2016, on Alepes kleinii*; 16 우, North Malabar (Ayikkara), 10.08.2017, on Euthynnus affinis; 6 오. North Malabar (Madakkara), 6.01.2018, on E. affinis; 42 우, Cochin (Kalamukku), 8.02.2016, on Scomberomorus commerson*; 17 ㅇ (Thoppumpadi), 10.02.2016, on $S$ commerson*

Records in India: Malabar (Calicut) [Thomas, 1967], on E. affinis; South Kerala (Vizhinjam) [Pillai, 1969], on E. affinis; North Malabar (Ayikkara) [Aneesh, 2014], on Mugil curema; South Kerala (Vizhinjam) [Silas, Ummerkutty 1967], on Sarda orientalis.

\section{Caligus constrictus Heller, 1865}

Material: 37 우, North Malabar (Ayikkara), 8.04.2014, on Alepes djedaba*t.

Records in India: Bombay [Rangnekar, 1956]; South Kerala (Vizhinjam) [Pillai, 1967], on Alectis indica, Carangoides malabaricus, and Stromateus sp.

Caligus cordyla Pillai, 1963

Material: 6 우, North Malabar (Ayikkara), 25.06.2015, on Megalaspis cordyla

Records in India: South Kerala (Vizhinjam) [Pillai, 1967]; North Malabar (Ayikkara) [Aneesh, 2014], on M. cordyla.

Caligus coryphaenae Steenstrup et Lütken, 1861

Material: 2 OP, North Malabar (Ayikkara), 4.12.2014, on Euthynnus affinis.

Records in India: India [Bassett-Smith, 1899; Kurian, 1961; Pillai, 1963a], on Acanthocybium solandri, Caranx hippos, C. melampygus, Coryphaena hippurus, Elagatis bipinnulata, Euthynnus affinis, E. alletteratus, Grammatorcynus bilineatus, Katsuwonus pelamis, Prionace glauca, Rachycentron canadum, Sarda orientalis, Seriola lalandi, Squalus acanthias, Thunnus albacares, T. obesus, and T. thynnus.

Caligus cybii Bassett-Smith, 1898

Material: 111 of , North Malabar (Ayikkara), 14.07.2018, on Scomberomorus commerson; 74 우, North Malabar (Ayikkara), 1.03.2016, on Euthynnus affinis*; 25 우, Cochin (Kalamukku), 9.06.2017, on E. affinis*; 10 우, Cochin (Thoppumpadi), 10.06 2017, on E. affinis*.

Records in India: South Kerala (Vizhinjam) [Pillai, 1967]; North Malabar (Ayikkara) [Silas, Ummerkutty, 1967, Chennai; Aneesh, 2014], on S. commerson, and S. lineolatus.

Caligus infestans Heller, 1865

Material: 1 \%, North Malabar (Ayikkara), 21.11.2014, on Scomberomorus commerson.

Records in India: India [Basset-Smith, 1898b, Pillai, 1969], on Euthynnus alletteratus and S. commerson.
Caligus kanagurta Pillai, 1961

Material:7 우, North Malabar (Ayikkara), 17.08.2017, on Rastrelliger kanagurta; 1 ㅇ, North Malabar (Madakkara), 28.08.2018, on R. kanagurta; 32 +9, Cochin (Thoppumpadi), 4.02.2016, on Decapterus russelli*.

Records in India: South Kerala (Vizhinjam) [Pillai, 1967]; South Kerala (Vizhinjam) [Silas, Ummerkutty, 1967]; North Malabar (Ayikkara) [Aneesh, 2014], on R. kanagurta.

Caligus longicaudatus Brady, 1899

Material: 6 우, Malabar, Chombala, 17.11.2016, on Leptomelanosoma indicum*.

Records in India: South Kerala (Vizhinjam) [BassetSmith, 1898a; Pillai, 1967d]; on Chirocentrus dorab and Trichiurus lepturus.

\section{Caligus pelamydis Krøyer, 1863}

Material: 16 우, North Malabar (Ayikkara), 26.10.2017, on Sarda orientalis*; 33 우, North Malabar (Ayikkara), 8.10.2014, on Euthynnus affinis*.

Caligus phipsoni Bassett-Smith, 1898

Material: $4 \mathrm{O}^{7} \mathrm{O}^{7}$, North Malabar (Ayikkara), 15.05.2015, on Scomberomorus commerson*.

Records in India: Bombay [Bassett-Smith 1898a]; South Kerala (Vizhinjam) [Pillai, 1963b]; on Eleutheronema tetradactylum, Plectropomus leopardus, Polydactylus plebeius, and Scomberomorus guttatus.

\section{Caligus priacanthi Pillai, 1961}

Material: 177 오, $1 \mathrm{O}^{7}$, North Malabar (chombala), 12.03.2016; 42 우, North Malabar (Azhikkal), 19.04.16; 6 우, North Malabar (Madakkara), 11.03.2019; 53 우 (Kalamukku), 26.10.2015; 11 우, Cochin (Thoppumpadi), 27.10.2015, all on Priacanthus hamrur.

Records in India: Bombay [Pillai, 1967], on P. hamrur.

Caligus regalis Leigh-Sharpe, 1930

Material: 7 우, North Malabar (Ayikkara), 21.05.2015, on Euthynnus affinis*.

Caligus rotundigenitalis Yü, 1933

Material:9 오우 $4 \bigcirc^{7} \sigma^{7}$. NorthMalabar (Thaikadappuram), 1.02.2018, on Caranx ignobilis*; 15 우, North Malabar (Madakkara), 8.03.2018, on C. ignobilis*; 205 OP, $17 \mathrm{O}^{7} \mathrm{O}^{7}$, North Malabar (Madakkara), 27.02.2018, on Etroplus suratensis*; 58 우, North Malabar (Madakkara), 27.02.2018, on Scatophagus argus; 32 우, Cochin (Kalamukku), 5.12.2018, on Mugil cephalus*.

Records in India: Bombay [Rangnekar 1959]; South Kerala [Pillai, Natarajan, 1977], on Gnathanodon speciosus, Lutjanus malabaricus and S. argus.

\section{Caligus savala Gnanamuthu, 1948}

Material: 9 우, Cochin (Kalamukku), 3.10.2016; 6 우, Cochin (Thoppumpadi), 15.10.2016, all on Trichiurus lepturus.

Records in India: South Kerala (Vizhinjam) [Gnanamuthu, 1948a, Chennai, Pillai, 1969], on Euthynnus affinis, Gaterin lineatus, Lepturacanthus savala, and T. lepturus.

\section{Caligus stromatei Krøyer, 1863}

Material:7 우, North Malabar (Ayikkara), 16.06.2017; 8 우, North Malabar (Azhikkal), 19.12.15; 110 우, Cochin (Kalamukku), 6.03.2017; 38 우, Cochin (Thoppumpadi), 6.03.2017, all on Pampus argenteus.

Previous records in India: South Kerala (Vizhinjam) [Pillai, 1967]; South Kerala (Vizhinjam) [Silas, Ummerkutty, 1967], all on P. argenteus. 
Caligus triangularis Shiino, 1954

Material: 6 우, North Malabar (Ayikkara), 4.12.2013, on Parajulis poecilepterus.

Previous records in India: North Malabar (Ayikkara) [Aneesh, 2014], on P. poecilepterus.

\section{Genus Euryphorus Milne Edwards, 1840}

Euryphorus nordmannii Milne Edwards, 1840

Material: 193 우, $52 \sigma^{7} \sigma^{7}$, North Malabar (Ayikkara), 6.03.2016; 225 OO, North Malabar (Azhikkal) 2.08.2015; 19 OO, $\mathrm{O}^{\top} \mathrm{O}^{\top}$, North Malabar (Madakkara), 5.12.2018; 128 우 (Kalamuk$\mathrm{ku}), 16.02 .2016 ; 42$ 우 , Cochin (Thoppumpadi), 16.02.2016, all on Coryphaena hippurus.

Records in India: South Kerala (Vizhinjam) [Pillai, 1964]; on C. equiselis, C. hippurus and Thunnus albacares.

\section{Genus Hermilius Heller, 1865}

Hermilius pyriventris Heller, 1865

Material: 102 우, North Malabar, 1.02.2018, on Netuma thalassina; 41 +P, Cochin (Kalamukku), 1.03.2017, on Arius acutirostris.

Records in India: South Kerala (Vizhinjam) [Pillai, 1963c], on A. acutirostris, Galeichthys feliceps, N. thalassina, and Plicofollis platystomus.

\section{Genus Parapetalus Steenstrup et Lütken, 1861}

Parapetalus hirsutus (Bassett-Smith, 1898)

Material: 6 우, Malabar, Chombala, 17.11.2016, on Leptomelanosoma indicum*; 22 우, North Malabar (Thaikadappuram), 28.09.2018, on Eleutheronema tetradactylum.

Records in India: South Kerala (Vizhinjam) [BassetSmith 1898a, Bombay; Pillai, 1962c], on E. tetradactylum and Polydactylus plebeius.

Parapetalus occidentalis Wilson C.B., 1908

Material: 23 우, Cochin (Kalamukku), 1.01.2016; 11 오, Cochin (Thoppumpadi), 14.01.2016, all on Rachycentron canadum

Records in India: South Kerala [Pillai, 1962a]; North Malabar (Ayikkara) [Aneesh, 2014], on Johnius sp. and $R$. canadum.

\section{Genus Synestius Steenstrup et Lütken, 1861}

Synestius caliginus Steenstrup et Lütken, 1861

Material: 371 कq, $13 \sigma^{7} \sigma^{7}$, North Malabar (Ayikkara), 23.03.2016, on Parastromateus niger $\$$

Records in India: South Kerala [Gnanamuthu 1950a, Chennai; Pillai, 1961]; North Malabar (Ayikkara) [Aneesh, 2014]; on Pampus argenteus and P. niger.

\section{Genus Tuxophorus Wilson C.B., 1908}

\section{Tuxophorus caligodes Wilson C.B., 1908}

Material: 3 우, North Malabar (Ayikkara), 3.12.2013 on Rachycentron canadumt.

Records in India: Bombay [Rangnekar, 1960]; South Kerala (Vizhinjam) [Pillai, 1961]; Echeneis naucrates, Tylosurus acus acus, Urophycis floridana; North Malabar (Ayikkara) [Aneesh, 2014], on R. canadum.

Tuxophorus cybii Nuñes-Ruivo et Fourmanoir, 1956

Material: $2 \sigma^{7} \sigma^{7}$, North Malabar (Ayikkara), 13.02.2015, on Scomberomorus commerson.
Records in India: South Kerala [Kurian, 1961], Acanthocybium solandri; North Malabar (Ayikkara) [Aneesh, 2014], on S. commerson.

Family Lernaeopodidae Milne Edwards, 1840 Genus Brachiella Cuvier, 1830

Brachiella thynni Cuvier, 1830

Material: 4 우, Malabar, Chombala, 19.12.2015, on Otolithes ruber*

Records in India: South Kerala (Vizhinjam) [Pillai, 1962d], on Acanthocybium solandri, Argyrosomus regius, Chirocentrus dorab, Pomatomus saltatrix, Sciaena umbra, Scomberomorus cavalla, S. lineolatus, Thunnus albacares, and Thunnus thynnus.

Brachiella trichiuri Gnanamuthu, 1951

Material: 2 우, North Malabar (Ayikkara), 25.09.2018, on Lepturacanthus savala; 16 우, North Malabar (Madakkara), 11.09.2018, on L. savala; 39 우, North Malabar (Ayikkara), 4.04.2016, on Trichiurus lepturus; 28 오, Cochin (Kalamukku), 10.12.2015, on T. lepturus; 12 우, Cochin (Thoppumpadi), 14.12.2015, on T. lepturus.

Records in India: South Kerala (Vizhinjam) [Gnanamuthu, 1951; Chennai; Pillai, 1968]; North Malabar (Ayikkara) [Aneesh, 2014]; on L. savala and T. lepturus.

\section{Genus Clavellisa Wilson, 1915}

Clavellisa dussumieriae Gnanamuthu, 1948

Material: 23 우, North Malabar (Ayikkara), 8.08.2015, on Sardinella fimbriata*

Records in India: South Kerala (Vizhinjam) [Gnanamuthu, 1947b; Chennai; Pillai, 1962b], on Dussumieria acuta, D. elopsoides; North Malabar (Ayikkara) [Aneesh, 2014], on D. elopsoides.

Clavellisa ilishae Pillai, 1962

Material: 54 우, North Malabar (Ayikkara), 7.10.2017, on Sardinella longiceps*; 88 우으, North Malabar (Ayikkara), 13.07.2015, on S. fimbriata*; 46 우, Cochin (Kalamukku), 23.03.2017, on S. fimbriata*.

Records in India: Odisha (Chilka lake) [Tripathi, 1962b]; Kolkatha (Hoogly) [Tripathi, 1962b]; South Kerala (Vizhinjam) [Pillai, 1962b]; North Malabar (Ayikkara) [Aneesh, 2014]; on Ilisha filigera and Tenualosa ilisha.

Clavellisa obcordatus Rangnekar, 1957

Material: 4 우, North Malabar (Ayikkara), 16.06.2017, on Thryssa mystax*; 8 오, North Malabar (Madakkara), 21.11.2018, on T. mystax*; 23 우, North Malabar (Ayikkara), 6.03.2016, on $T$. setirostris*; 12 우, North Malabar (Azhikkal), 2.08.2015, on $T$. setirostris*; 42 우, North Malabar (Ayikkara), 13.10.2015, on T. malabarica; 16 우 North Malabar (Azhikkal), 2.08.2015, on $T$. malabarica; $170 \bigcirc \bigcirc$, Cochin (Kalamukku), 26.03.2015, on T malabarica, 32 우, Cochin (Kalamukku), 21.04.2015, on Pellona ditchela*.

Records in India: Bombay [Rangnekar, 1957a]; South Kerala (Vizhinjam) [Pillai, 1962b], on Nematalosa nasus and $T$. malabarica.

Clavellisa phasa Tripathi, 1962

Material: 118 oㅇ, North Malabar (Ayikkara), 4.03.2015, on Coilia dussumieri.

Records in India: Kolkatha (Hoogly) [Tripathi, 1962b], on C. dussumieri and Setipinna phasa. 
Genus Naobranchia Hesse, 1863

Naobranchia cygniformis Hesse, 1863

Material: 11 우, North Malabar (Ayikkara), 3.12.2015, on Anodontostoma chacunda*.

Naobranchia vermiformis Rangnekar, 1956

Material: 2 우, North Malabar (Ayikkara), 27.01.2018; 18 우, Cochin (Kalamukku), 3.12.2018; 11 웅, Cochin (Thoppumpadi), 26.09.2017, all on Rastrelliger kanagurta*.

Records in India: Bombay [Rangnekar, 1956]; North Malabar (Ayikkara) [Aneesh, 2014], on Tenualosa toli.

\section{Genus Parabrachiella Wilson C.B., 1915}

Parabrachiella albida (Rangnekar, 1956)

Material: 5 우, North Malabar (Ayikkara), 3.08.2017; 20 우, North Malabar (Madakkara), 11.09.2018; 55 오, Cochin (Kalamukku), 3.08.2015; 15 우, Cochin (Thoppumpadi), 3.08.2015, all on Otolithes ruber.

Records in India: Bombay [Rangnekar, 1956]; Odisha [Tripathi, 1962a]; South Kerala (Vizhinjam) [Pillai, 1968d]; on Johnius glaucus and O. ruber.

Parabrachiella otolithi (Pillai, 1962)

Material: 2 우, Cochin (Kalamukku), 23.03.2017, on Otolithes cuvieri*.

Records in India: South Kerala (Vizhinjam) [Pillai, 1967d], on O. ruber.

Genus Thysanote Krøyer, 1863 1861)

Thysanote appendiculata (Steenstrup et Lütken,

Material: 16 o+, North Malabar (Ayikkara), 14.04.2014, on Parastromateus niger $\$$.

Records in India: Bombay [Basset-Smith, 1898a]; South Kerala (Vizhinjam) [Gnanamuthu, 1950c, Chennai; Pillai, 1967]; North Malabar (Ayikkara) [Aneesh, 2014]; on $P$. niger.

Thysanote eleutheronema Rangnekar, 1961

Material: 2 우, North Malabar (Madakkara), 28.09.2018, on Eleutheronema tetradactylum.

Records in India: Bombay [Rangnekar, 1961]; Kolkatha [Tripathi, 1962b]; South Kerala (Vizhinjam) [Pillai, 1967d], on E. tetradactylum and Polydactylus plebeius.

Family Lernanthropidae Kabata, 1979

Genus Lernanthropinus Ho et Do, 1985

Lernanthropinus decapteri (Pillai, 1964)

Material: 19 우, North Malabar (Ayikkara), 4.11.2015, on Decapterus russelli; 24 오, North Malabar (Azhikkal) 2.08.2015, on D. russelli; 126 우, Cochin (Kalamukku), 29.09.2015, on Thryssa malabarica*.

Records in India: South Kerala (Vizhinjam) [Pillai, 1967]; North Malabar (Ayikkara) [Aneesh, 2014], all on D. russelli.

Lernanthropinus forficatus (Redkar, Rangnekar et Murti, 1949)

Material: 4 오, North Malabar (Ayikkara), 26.10.2017, on Lepturacanthus savala; 93 우, North Malabar (Ayikkara), 13.03.2016, on Trichiurus lepturus; 24 우, Cochin (Kalamukku), 21.10.2015, on T. lepturus; 16 우, Cochin (Thoppumpadi), 14.12.2015, on T. lepturus.
Records in India: Bombay [Redkar et al., 1949]; South Kerala (Vizhinjam) [Pillai, 1967d], on Lepturacanthus savala and T. lepturus.

Lernanthropinus sphyraenae (Yamaguti \& Yamasu, 1959)

Material: 49 우, North Malabar (Azhikkal), 19.12.2015, on Mene maculata ; 200 우, $12 \sigma^{7} \sigma^{7}$, North Malabar (Ayikkara), 7.10.2015, on M. maculata; 220 우, Cochin (Kalamukku), $21.10 .2015 ; 199$ 우, Cochin (Thoppumpadi), 27.07.2015, on M. maculata $\ddagger$.

Lernanthropinus temminckii (von Nordmann, 1864)

Material: 17 OP. North Malabar (Ayikkara), 11.10.2017, on Saurida tumbil; 40 우, North Malabar (Ayikkara), 3.11.2015, on S. undosquamis*;; 76 우, North Malabar (Azhikkal), 13.12.2015, on S. undosquamis*.

Records in India: South Kerala (Vizhinjam) [Pillai, 1967], on S. tumbil.

Genus Lernanthropus de Blainville, 1822

Lernanthropus abitocephalus Tripathi, 1962

Material: 6 of , North Malabr (Ayikkara), 27.01.2014, on $\mathrm{Ni}-$ bea maculata*.

Records in India: Odisha (Puri) [Tripathi, 1962a]; Bombay [Rangnekar, Murti, 1961]; on Pomadasys maculatus.

Lernanthropus belones Krøyer, 1863

Material: 976 웅, $92 \sigma^{7} \sigma^{7}$, North Malabar (Azhikkal), 1.10.2014, on Ablennes hians* $\$ ; 200$ 우, 52 ऽ $^{\top} \sigma^{7}$, North Malabar (Azhikkal), 15.03.2015, on Strongylura leiura*ł.

Lernanthropus brevicephalus Rangnekar, 1957

Material: 19 우, Cochin (Thoppumpadi), 9.02.2016, on Lutjanus sanguineus*.

Records in India: South Kerala (Vizhinjam) [Rangnekar, 1957, Bombay, Pillai, 1967], on Elops senegalensis and Megalops cyoprinoides.

Lernanthropus cadenati Delamare Deboutteville et Nunes-Ruvio, 1954

Material: 33 우, North Malabar (Azhikkal), 19.07.2013, on Megalops cyprinoides.

Records in India: South Kerala (Vizhinjam) [Pillai, 1967], on Elops senegalensis and M. cyprinoides.

Lernanthropus corniger Yamaguti, 1954

Material: 136 우, $10^{7}$, North Malabar (Ayikkara),3.05.2015,on Megalaspis cordyla $\$$; 89 우으, North Malabar (Madakkara), 6.01.2018, on M. cordyla; 26 우, North Malabar (Thaikadappuram), 21.02.2018, on M. cordyla; 86 오, Cochin (Kalamukku), 20.08.2015, on Alepes djedaba*\$; 124 우, Cochin (Kalamukku), 20.08 .2015 , on M. cordyla; 68 우, Cochin (Thoppumpadi), 2.06.2015, on M. cordyla.

Records in India: South Kerala (Vizhinjam) [Pillai, 1967]; Odisha (Puri) [Tripathi, 1962a]; North Malabar (Ayikkara) [Aneesh, 2014]; on M. cordyla and Rastrelliger kanagurta.

Lernanthropus dussumieria Gnanamuthu, 1949

Material: 17 우, Cochin (Kalamukku); 11 우, Cochin (Thoppumpadi), 11.11.2015, all on Dussumieria acuta.

Records in India: South Kerala (Vizhinjam) [Gnanamuthu, 1948b, Chennai; Pillai, 1967], on D. acuta; North Malabar (Ayikkara) [Aneesh, 2014], on Scomberoides tol. 
Lernanthropus giganteus Krøyer, 1863

Material: 88 우, 1 ᄋ , North Malabar (Ayikkara), 7.10.2017, on Alepes djedaba

Lernanthropus ilishae Chin, 1948

Material: 8 우, North Malabar (Ayikkara), 23.05.2015, on Opisthopterus tardoore*.

Lernanthropus indicus Pillai, 1967

Material: 1 + , North Malabar (Ayikkara), 25.06.2013, on Caranx sexfasciatus $*$.

Records in India: South Kerala (Vizhinjam) [Pillai, 1967], on C. ignobilis.

Lernanthropus koenigii Steenstrup et Lütken, 1861 Material: 4 우, North Malabar (Ayikkara), 18.02.2014 on Carangoides coeruleopinnatus $*+$.

Records in India: Chennai [Gnanamuthu, 1950b]; North Malabar (Ayikkara) [Aneesh, 2014]; on Parastromateus niger and Peprilus paru.

Lernanthropus latis Yamaguti, 1954

Material: 1 +, Cochin (Kalamukku), 23.03.2017, on Otolithes cuvieri*

Records in India: Odisha and Bengal [Tripathi, 1962a], on Lates calcarifer.

\section{Lernanthropus leiognathi Tripathi, 1962}

Material: 440 우, $2 \bigcirc^{\top} \bigcirc^{7}$, North Malabar (Azhikkal), 23.05. 2015, on Eubleekeria splendens*; 4 우, North Malabar (Azhikkal), 25.04.2013, on Equulites elongatus*; 8 우, North Malabar (Azhikkal), 28.10.2013, on Leiognathus brevirostris*; 23 우, North Malabar (Azhikkal), 30.10.2013, on L. daura; 10 우, North Malabar (Azhikkal), 12.07.2013, on L. equulus*; 1 \%, North Malabar (Azhikkal), 12.08.2013, on L. fasciatus*; 7 우, North Malabar (Azhikkal), 12.08.2013, on Nuchequula blochii*; 19 우, North Malabar (Azhikkal), 25.04.2013, on Photopectoralis bindus*.

Records in India: Odisha [Tripathi, 1962a], on L.s daura and Secutor ruconius.

Lernanthropus opisthopteri Pillai, 1964

Material: 10 우, North Malabar (Ayikkara), 13.07.2017, on Opisthopterus tardoore.

Records in India: South Kerala (Vizhinjam) [Pillai, 1967], on $O$. tardoore.

Lernanthropus otolithi Pillai, 1963

Material: 13 우, North Malabar (Azhikkal), 19.12.2015; 22 오, North Malabar (Madakkara), 18.04.2018; 62 우, Cochin (Kalamukku), 28.10.2015; 53 우, Cochin (Thoppumpadi), 2.11.2015, all on Otolithes ruber.

Records in India: South Kerala (Vizhinjam) [Pillai, 1967], on O. ruber and Pterotolithus maculatus.

Lernanthropus polynemi Richiardi, 1881

Material: 3 우, North Malabar (Madakkara), 28.09.2018, on Eleutheronema tetradactylum.

Records in India: Odisha (Puri) [Tripathi, 1962a]; South Kerala (Vizhinjam) [Pillai, 1966], on E. tetradactylum.

Lernanthropus sciaeni Gnanamuthu, 1947

Material: 2 of , North Malabar (Azhikkal), 22.06.2013, on Johnius trachycephalus*; 15 우, Cochin (Thoppumpadi), 23.03. 2017, on J. glaucus.

Records in India: South Kerala (Vizhinjam) [Gnanamuthu, 1947a; Chennai; Pillai, 1967], on J. glaucus.

\section{Lernanthropus secutoris Pillai, 1963}

Material: 42 우, North Malabar (Ayikkara) 30.10.2013, on Secutor insidiator; 5 우, North Malabar (Ayikkara), 21.01.2014, on S. ruconius*.

Records in India: South Kerala (Vizhinjam) [Pillai, 1967], on $S$. insidiator.

\section{Lernanthropus sillaginis Pillai, 1963}

Material: 17 우, North Malabar (Madakkara), 18.04.2018; 9 우 , North Malabar (Thaikadappuram) 24.02.2018, all on Sillago sihamał.

Records in India: South Kerala (Vizhinjam) [Pillai, 1967], on S. sihama.

\section{Lernanthropus triangularis Pillai, 1963}

Material: 1 + , North Malabar (Ayikkara), 12.07.2013, on $\mathrm{Ar}$ gyrops filamentosus* $; ; 1$ +, North Malabar (Ayikkara), 23.10.2014, on Gerres erythrourus*t.

Records in India: South Kerala (Vizhinjam) [Pillai, 1963d], on Gerres filamentosus.

Lernanthropus tylosuri Richiardi, 1880

Material: 154 우, 9 Ơ $^{\top}$, North Malabar (Ayikkara), 4.11.2017, on Strongylura leiura ; 39 우, North Malabar (Ayikkara), 17.04.2016, on S. strongylura; 79 우, Cochin (Kalamukku), 18.6.2015, on Hemiramphus lutkei*!; 95 우, Cochin (Kalamuk$\mathrm{ku}), 3.08 .2015$, on S. strongylura.

Records in India: South Kerala (Vizhinjam) [Pillai, 1963d], on Ablennes hians, Platybelone argalus argalus, Strongylura anastomella, S. exilis, S. incisa, S. leiura, S. marina, S. strongylura, S. timucu, S. urvillii, Tylosurus acus acus, T. choram, T. crocodilus crocodilus, and T. punctulatus; North Malabar (Ayikkara) [Aneesh, 2014], on S. leiura, $S$. strongylura, and T. crocodilus crocodilus.

Lernanthropus villiersi Delamare Deboutteville et Nunes-Ruivo, 1954

Material: 2 우, North Malabar (Ayikkara), 18.07.2013, on Rhynchopelates oxyrhynchus*.

\section{Genus Mitrapus Song et Chen, 1976}

Mitrapus engraulis (Tripathi, 1962)

Material: 10 우, North Malabar (Ayikkara), 18.12.2014, on Stolephorus baganensis*; 8 우, North Malabar (Ayikkara), 21. 02.2015, on S. tri*.

Records in India: Kolkatha, Hoogly [Tripathi, 1962a], on Setipinna phasa.

Mitrapus oblongus (Pillai, 1964)

Material: 5 오, North Malabar (Ayikkara), 29.06.2017, on Scolopsis bimaculata*; 3 우, North Malabar (Madakkara), 24.05. 2018, on Sardinella fimbriata; 6 o+, North Malabar (Ayikkara), 21.11.2018, on S. fimbriata; 39 +P, Cochin (Kalamukku), 23.03. 2017, on S. fimbriata.

Records in India: South Kerala (Vizhinjam) [Pillai, 1967]; North Malabar (Ayikkara) [Aneesh, 2014], all on $S$. fimbriata.

\section{Genus Norion von Nordmann, 1864}

Norion priacanthi (Kirtisinghe, 1956)

Material: 387 우, $4 \bigcirc^{7} \sigma^{7}$, North Malabar (Ayikkara), 12.03. 2016; 83 우, North Malabar (Madakkara), 20.09.2018; 166 우, Cochin (Kalamukku), 26.10.2015; 90 우, Cochin (Thoppumpadi), 26.10.2015; all on Priacanthus hamrur. 
Records in India: South Kerala (Vizhinjam) [Pillai, 1967], on $P$. hamrur.

\section{Genus Sagum Wilson C.B., 1913}

Sagum epinepheli (Yamaguti et Yamasu, 1960)

Material: 114 +O, $2 \mathrm{O}^{7} \mathrm{O}^{7}$, North Malabar (Ayikkara), 3.11.2015, on Epinephelus diacanthus* $*$.

Records in India:, South Kerala (Vizhinjam) [Pillai, Sebastian 1967], on E. akaara and E. merra.

Family Pennellidae Burmeister, 1835

Genus Lernaeenicus Lesueur, 1824

Lernaeenicus stromatei Gnanamuthu, 1953

Material: 151 oㅇ, North Malabar (Ayikkara), 10.02.2016, on Parastromateus nigert. niger.

Records in India: Chennai [Gnanamuthu, 1953], on $P$.

Genus Peniculus Nordmann, 1832

Peniculus fistula fistula Nordmann, 1832

Material: 37 우, North Malabar (Ayikkara), 8.11.2015, on Anodontostoma chacunda*.

Family Pseudocycnidae Wilson C.B., 1922

Genus Cybicola Bassett-Smith, 1898

Cybicola armatus (Bassett-Smith, 1898)

Material: 1106 우, North Malabar (Ayikkara), 1.03.2016; 2 우, North Malabar (Madakkara), 2.10.2018; 1 ㅇ, North Malabar (Thaikadappuram), 21.02.2018; 254 우, Cochin (Kalamukku), 25.06.2015; 133 우 (Thoppumpadi), 16.07.2015; all on Scomberomorus commerson.

Records in India: Bombay [Bassett-Smith, 1898b]; South Kerala (Vizhinjam) [Gnanamuthu, 1950b; Chennai, Pillai, 1964]; North Malabar (Ayikkara) [Silas, Ummerkutty, 1967; Aneesh, 2014]; on S. commerson, S. guttatus, and S. queenslandicus.

\section{Genus Pseudocycnus Heller, 1865}

Pseudocycnus appendiculatus Heller, 1865

Material: 35 우, $3 \bigcirc^{7} \sigma^{7}$, North Malabar (Ayikkara), 8.08.2014, on Thunnus albacares; 12 오, North Malabar (Ayikkara), 28.04.2014, on T. tonggol*; 185 +o+, $4 \mathrm{O}^{\top} \mathrm{O}^{\mathrm{T}}$, North Malabar (Ayikkara), 1.03.2016, on Euthynnus affinis; 16 오, $4 \bigcirc^{7} \sigma^{7}$, North Malabar (Madakkara), 6.01.2018, on E. affinis; 67 우 (Kalamuk$\mathrm{ku}), 28.06 .2015$, on E. affinis; 23 우, Cochin (Thoppumpadi), 10.11.2015, on E. affinis.

Records in India: South Kerala (Vizhinjam) [Pillai, 1964]; North Malabar (Ayikkara) [Aneesh, 2014]; on E. affinis, E. alletteratus, Katsuwonus pelamis, Sarda sarda, Thunnus alalunga, T. albacares, T. obesus, and T. thynnus.

\section{Comments}

This study of copepods living on the marine fish along the Kerala coast of India records a great diversity of parasitic copepods, several of which were previously unknown in the region. The impact of these parasites on their hosts remains unknown [Kabata, 1985]. The study also found a number of marine fishes that were previously unknownon the Indian coastas hosts of copepods. A comparison of the obtained data with that of the great diversity of fish living in the coastal waters of India, until now mostly unexplored for parasites, indicates the potential presence in India's waters of an unexplored diversity of parasitic copepods. A more thorough study is needed of the diversity and ecology of parasitic copepods, both in the area studied and in other areas of the Indian coast, most of which remain unexplored. In addition, a thorough study of parasitic copepods and other invertebrates living on fishes is necessary to provide a better understanding of the potential impact of these parasites on commercially important fish and the marine environmentand to assess the diversity and potential role of the parasitic copepods in the functioning of coastal communities around India, especially because these communities are rapidly changing under the conditions of the anthropogenic stress, pollution or climate change observed in the past years [Ramýrez et al., 2017].

Taking the above into account, it seems that the most promising direction for further study of the copepods parasitizing fish and invertebrates around India and the adjacent regions is the pursuit of an integrative approach to the study of the diversity of parasitic copepods, their phylogeography and their specificity to hosts [Ivanenko et al., 2018]. Of particular importance in conducting such an investigation are the monitoring of the abundant diversity of parasitic copepods living on fish and invertebrates in various regions of India and the creation and maintenance of a collection of copepods and, equally important, tissue samples of their hosts that can be studied by molecular and morphological methods.

Acknowledgements. The research work of SK was funded by DST-SERB research project (No. EMR/2016/001163/ AS, 28 August 2017) and DST-RFBR collaborative research project (No. INT/RUS/RFBR/P-330, 10.01.2019). The research of NRMV was funded by DST (DST/INSPIRE Fellowship/2017/IF170696, 04.07.2018). The technical support was provided by Dr. Priya Jose and Mr. P. Swaraj. The research of VNI was funded by RFBR according to the research project No. 18-54-45016 IND_a. Frank Ferrari commented on a draft of the manuscript; Oksana Korzhavina (Lomonosov Moscow State University) helped to prepare the map.

Authors contributions. NRMV, DOK, RK and MTMV, HAK collected and identified the specimens. SK initiated and supervised the project as well as took part in preparation of the manuscript. VNI took part in planning and preparation of the manuscript.

\section{References}

Aneesh P.T. 2014. Studies on parasitic crustaceans infesting the fishes of Malabar Coast // Ph. D Thesis. Kannur University. P.1-147.

Aneesh P.T., Helna A.K., Kumar A.B., Maran B.A.V. 2018. Redescription of LernaeenicusstromateiGnanamuthu, 1953 (Copepoda: Siphonostomatoida: Pennellidae) infesting the black pomfret Parastromateusniger (Bloch) from Indian waters // Zootaxa. Vol.4482. No.2. P.375-382. 
Aneesh P.T., Sudha K., Helna A.K., Anilkumar G., Trilles J.P. 2014. Multiple parasitic crustacean infestation on belonid fish, Strongylurastrongylura // ZooKeys. Vol.457. P.339-353.

Aneesh P.T., Sudha K., Helna A.K., Arshad K., Anilkumar G. Trilles J.P. 2013. Simultaneous multiple parasitic crustacean infestation on banded needlefish, Strongylura leiura (Belonidae) from the Malabar Coast, India // International Journal of Scientific and Research Publications. Vol.3. No.7. P.367-375.

Bassett-Smith P.W. 1898a. I. Some new parasitic Copepods found on fish at Bombay // Journal of Natural History.Vol.1. No.1. P.1-17.

Bassett-Smith P.W. 1898b. Some new or rare parasitic copepods found on fish in the Indo-Tropical region // Annals and Magazine of Natural History. Ser.7. Vol.7. P.357-372.

Bassett-Smith P.W. 1899. A systematic description of parasitic copepoda found on fish with an enumeration the known species // Proceedings of the Zoological Society of London. Vol.67. No.2. P.438-507.

Bennet P.S. 1964. On Bomolochus sardinellae sp. nov. (Copepoda: Cyclopoida) parasitic on Sardinella albella // Journal of the Marine Biological Association of India. Vol.6. 84-88.

Bennet P.S. 1966. Bomolochus varunae a new species of parasitic copepod from Anodontostoma chacunda // Journal of the Marine Biological Association of India. Vol.8. No.2. P.295-301.

Bennet P.S. 1967. On Bomolochus jonesi sp. nov. parasitic on the eye of the Indian mackerel Rastrelliger kanagurta // Journal of the Marine Biological Association of India. Vol.9. No.1. P.132136.

Froese R., Pauly D. (eds.). 2019. FishBase. World Wide Web electronic publication // www.fishbase.org. (02/ 2019).

Gnanamuthu C.P. 1947a. Lernanthropus sciaenae sp. nov. a copepod parasitic on the gills of Sciaena glauca from Madras // Records of Indian Museum. Vol.45. P.291-298.

Gnanamuthu C.P. 1947b. A new copepod parasite Clavellisa dussumieriae belonging to the subfamily Ñlavellinae from the gills of a Madras fish // Proceedings of the Zoological Society of London. Vol.117. No.4. P.748-755.

Gnanamuthu C.P. 1947c. Bomolochus multispinosa sp. nov. and ergasilid copepod observed in copulation // Records of Indian Museum. Vol.45. P.309-319.

Gnanamuthu C. P. 1948a. Notes on the anatomy and physiology of Caligus savala n.sp., a parasitic copepod from Madras plankton // Proceedings of the Zoological Society of London. Vol.118. No.3. P.591-606.

Gnanamuthu C.P. 1948b. A new copepod parasite Lernanthropus dussumieria n.sp. from the gills of a marine fish // Parasitology. Vol.39. No.3\&4. P.209-213

Gnanamuthu C.P. 1949. Two male parasitic copepods from Madras // Annals and Magazine of Natural History. Ser.12. Vol.2. No.17. P.359-367.

Gnanamuthu C.P. 1950a. Synestius caliginus Steenstrup \& Lutken a copepod parasite of the grey pomfret // Records of the Indian Museum. Vol.47. P.253-258.

Gnanamuthu C.P. 1950b. Two dichelesthid copepods from Madras fish // Parasitology. Vol.40. No.3\&4. P.276-282.

Gnanamuthu C.P. 1950c. Thysanote appendiculata (Steenstrup and Lutken) lernaeopodid parasitic on the gills of the grey pomfret // Records of the Indian Museum. Vol.47. P.259-264.

Gnanamuthu C.P. 1951. Brachiella trichiuri n. sp. a copepod parasite in mouth cavity of the ribbon fish // Spolia zeylanica. Vol.26. P.13-15.

Gnanamuthu G.P. 1953. Three lernaeid copepods parasitic on South Indian fishes // Journal of Parasitology. Vol.39. No.1. P.1-8.

Hameed S.M. 1976. Description of a new species of Anuretes (Copepoda: Caligidae) from Kerala // Hydrobiologia. Vol.51. No.1. P.39-42.

Hammed S.M., Pillai N.K. 1973. Description of a new species of Caligus (Crustacea: Copepoda) from Kerala // Zoological Anzeiger. Vol.191. P.114-118.

Helna A.K., Aneesh P.T., Arshad K., Sudha K. 2013.Occurrence of Caligus sp. a parasitic copepod (Crustacea) infesting the commercially exploited fishes along the Malabar cost // Journal of Basic and Applied Biology. Vol.7. No.3. P.28-30.
Helna A.K., Sudha K., Aneesh P.T., Anilkumar G. 2018. Caligus cybii (Caligidae, Copepoda) parasitising the commercially exploited seer fish, Scomberomorus commerson, from the Malabar Coast (India) - occurrence and adaptations // Turkish Journal of Fisheries and Aquatic Sciences. Vol.18. No.3. P.445-455.

Helna A.K., Sudha K., Aneesh P.T., Piasecki W., Anilkumar G. 2016. A case of persisting massive infection of Scomberomorus commerson, a commercially exploited scombrid fish, with Cybicola armatus (Copepoda: Siphonostomatoida: Pseudocycnidae) // Acta parasitologica. Vol.61. No.4. P.836-848.

Ho J.S., Jayarajan P., Radhakrishnan S. 1992. Copepods of the family Ergasilidae (Poecilostomatoida) parasitic on coastal fishes of Kerala, India // Journal of Natural History. Vol.26. No.6. P.1227-1241.

Ivanenko V.N., Hoeksema B.W., Mudrova S.V., Nikitin M.A., Martínez A., Rimskaya-Korsakova N.N., Berumen M.L., Fontaneto D. 2018. Lack of host specificity of copepod crustaceans associated with mushroom corals in the Red Sea // Molecular Phylogenetics and Evolution. Vol.127. P.770-780.

Kabata Z. 1985. Parasites and diseases of fish cultured in the tropics. London: Taylor \& Francis Ltd. 318 p.

Kirtisinghe P. 1964. A review of the parasitic copepods of fish recorded from Ceylon with description of additional forms // Bulletin of the Fisheries Research Station, Ceylon. Vol.17. No.1. P.45-132.

Kurian C.V. 1955. Parasitic copepods of fishes from Travancore Cochin // Bulletin of Research Institute, University of Travancore. Vol.4. P.103-116.

Kurian C.V. 1961. Parasitic copepods of fishes from Kerala // Bulletin of Research Institute, University of Kerala. Vol.8. P.63-77.

Pillai N.K. 1961. Copepods parasitic on south Indian fishes. Pt. 1. Caligidae // Bulletin of Research Institute, University of Kerala. Vol.8. P.87-130.

Pillai N.K. 1962a. Revision of the genera Parapetalus Steenstrup \& Lutken and Pseudopetalus nov. // Crustaceana. Vol.3. 285303.

Pillai N.K. 1962b. Copepods parasitic on South Indian Fishes. Families Lernaneopodidae and Naobranchidae // Journal of the Marine Biological Association of India. Vol.4. 58-94.

Pillai N.K. 1963a. Observations on the synonymy of Caligus coryphaenae Stp. \& Lutk. // Annals and Magazine of Natural History. Ser.13. Vol.13. No.57. P.513-522.

Pillai N.K. 1963b. Copepods parasitic on South Indian fishes. Family Caligidae // Journal of the Marine Biological Association of India. Vol.5. P.68-96.

Pillai N.K. 1963c. A revision of the genus Hermilius (CopepodaCaligidae) // Journal of the Zoological Society of India. Vol.14. P.179-187.

Pillai N.K. 1963d. Copepods parasitic on South Indian fishes. Family Anthosomidae I // Journal of Bombay Natural History Society. Vol.60. P.657-670.

Pillai N.K. 1964. A miscellaneous collection of copepods parasitic on south Indian fishes // Journal of the Marine Biological Association of India. Vol.6. No.1. P.61-83.

Pillai N.K. 1966. Notes on copepods parasitic on South Indian marine fishes // Journal of the Marine Biological Association of India. Vol.8. P.123-140.

Pillai N.K. 1967. Copepods parasitic on Indian marine fishes. A review // Proceedings of Symposium on Crustacea, Cochin. Vol.5. P.1556-1680.

Pillai N.K. 1968. Description of some species of Brachiella and Clavellopsis with comments on Isobranchia Heegaard // Crustaceana. Vol.1. P.119-135.

Pillai N.K. 1969. Notes on some copepod parasites in the collection of the British Museum (N.H.) London // Journal of the Marine Biological Association of India. Vol.11. No.1\&2. P.149-174.

Pillai N.K. 1970. A few examples showing the effect of host on the morphological characters of copepod parasites // Journal of Parasitology. Vol.56. P.267.

Pillai N.K. 1971. On the transfer of Bomolochus varunae Bennet to Pseudorbitacolax gen. nov. (Copepoda Bomolochidae) // Journal of the Zoological Society of India. Vol.23. P.13-19. 
Pillai N.K. 1973. Three new bomolochids parasitic on fishes of the Kerala coast // Indian journal of fisheries. Vol.20. No.2. P.487496.

Pillai N.K. 1985. The fauna of India: Copepod parasites of marine fishes // Zoological Survey of India. P.1-814.

Pillai N.K., Natarajan P. 1977. Copepods parasitic on fishes of the Kerala coast // Aquatic Biology. Vol.2. P.19-43.

Pillai N.K., Sebastian M.J. 1967. Redescription of Sagum epinepheli (Yamaguti\&Yamasu) with comments on the validity of Pseudolernanthropus (Copepoda Anthosomatidae) // Crustaceana. Vol.13. P.73-80.

Ramýrez F., Afan I., Davis L.S., Chiaradia A. 2017. Climate impacts on global hot spots of marine biodiversity // Science Advances. Vol.3. No.2. e1601198.

Rangnekar M.P. 1956. Parasitic copepods from marine fishes of Bombay // Journal of University of Bombay. Vol.24. P.42-65.

Rangnekar M.P. 1957. Copepod parasites of the family Argulidae, Caligidae, Dichestilidae and Lernaeopodidae // Journal of University of Bombay. Vol.26. No.3. P.8-20.

Rangnekar M.P. 1959. Parasitic copepods from fishes of the western coast of India with description of one new and redescription of four known species // Journal of the University of Bombay. Vol.28. P.43-58.

Rangnekar M.P. 1960. Tuxophorus caligodes Wilson a copepod parasitic on a local fish // Journal of Biological Sciences. Vol.3. 20-25.

Rangnekar M.P. 1961. Copepods parasitic on fishes of Bombay. 1 Lernaeopodida // Journal of University of Bombay.Vol.29. P.193-205.

Rao T.S. 1950. On a new caligid parasite from the Indian hammerhead shark // Proceedings of the Indian Academy of Sciences SectionB. Vol.31. No.6. P.302-307.

Redkar M.V., Rangnekar P.G., Murti N.N. 1949. Four new species of parasitic copepods from the marine fishes of Bombay // Journal of University of Bombay. Vol.18. P.36-50.

Rijin K., Sudha K., Vineesh P.J., Anilkumar G. 2018. Seasonal variation in the occurrence of parasitic isopods and copepods (Crustacea) infecting the clupeidaen fishes of Malabar coast, India // Turkish Journal of Fisheries and Aquatic Sciences. Vol.19. No.3. P.241-249.

Santhosh B. 2000. Metazoan parasites of mullets of Kerala coast: Taxonomy and community characteristics. Ph.D Thesis, University of Kerala.
Sebastian M.J. 1964. Taeniacanthus dentatus sp. nov. a copepod parasite of the fish Bembrops caudimaculata Steindachner // Journal of the Marine Biological Association of India. Vol.6. 94-97.

Sebastian M.J. 1966. On a new species of Lernaeenicus, L. bataviensis (Copepoda - Lernaeidae) with a key for the identification of the Indian species // Journal of the Marine Biological Association of India. Vol.1. P.114-118.

Sebastian M.J., George K.C. 1964. Lernaeenicus anchoviellae n. sp. (Copepoda - Lernaeidae) parasitic on Anchoviella bataviensis (Hardenberg) with descriptions of its three post-larval stages // Journal of the Marine Biological Association of India. Vol.6. No.2. P.235-240.

Silas E.G., Ummerkutty A.N.P. 1967. Parasites of scombroid fishes Part II: Parasitic copepod // Symposium on scomberoid fishes, India. Vol. 3. P.876-993.

Thomas M.M. 1967. A new parasitic copepod, Caligus krishnai, from the mackerel tuna Euthynnus affini saffinis (Cantor) // Journal of the Marine Biological Association of India. Vol. 9. No.1. P.137-140.

Tripathi Y.R. 1960. Parasitic copepods from Indian fishes. 1. Family Chondracanthidae Milne Edwards 1840 // Journal of the Zoological Society of India. Vol.12. P.51-59.

Tripathi Y.R. 1962a. Parasitic copepods from Indian fishes. III. Family Anthosomatidae and Dishelestiidae // Proceedings of the first All-Indian Congress of Zoology. Vol 2. P.191-217.

Tripathi Y.R. 1962b. Parasitic copepods from Indian fishes. VI Achtheriformes // Proceedings of the first All-Indian Congress of Zoology. Vol.2. P.218-233.

Vinoth R., Ajith Kumar T.T., Ravichandran S., Gopi, M., Rameshkumar G. 2010. Infestation of nopepod parasites in the food fishes of Vellar estuary, southeast coast of India // Acta Parasitologica Globalis. Vol.1. No.1. P.1-5.

WoRMS. 2019. World Register of Marine Species. Available from http://www.marinespecies.org at VLIZ. Accessed 2019-06-02. doi: $10.14284 / 170$

Yamaguti S. 1963. Parasitic Copepoda and Branchiura of fishes. New York: Interscience Publishers. Vol.1\&2. 1104 p.

Responsible editor K.G. Mikhailov 